\title{
Mars Exploration Rover Surface Operations: Driving Spirit at Gusev Crater
}

\author{
P. Chris Leger, Ashitey Trebi-Ollennu, John R. Wright, Scott A. Maxwell, Robert G. Bonitz, \\ Jeffrey J. Biesiadecki, Frank R. Hartman, Brian K. Cooper, Eric T. Baumgartner, and Mark W. Maimone \\ Jet Propulsion Laboratory, California Institute of Technology \\ Pasadena, CA, USA \\ chris.leger@jpl.nasa.gov
}

\begin{abstract}
Spirit is one of two rovers that landed on Mars in January 2004 as part of NASA's Mars Exploration Rover mission. As of July 2005, Spirit has traveled over 4.5 kilometers across the Martian surface while investigating rocks and soils, digging trenches to examine subsurface materials, and climbing hills to reach outcrops of bedrock. Originally designed to last $90 \mathrm{sols}$ (Martian days), Spirit has survived over 500 sols of operation and continues to explore. During the mission, we achieved increases in efficiency, accuracy, and traverse capability through increasingly complex command sequences, growing experience, and updates to the on-board and ground-based software. Safe and precise mobility on slopes and in the presence of obstacles has been a primary factor in development of new software and techniques.
\end{abstract}

Keywords: Planetary robotics, mobility, MER, Mars, rovers.

\section{Introduction}

NASA's Mars Exploration Rover (MER) mission developed and operates two robotic vehicles tasked with searching for evidence of past water activity on Mars [1]. The $175 \mathrm{~kg}, 1.6 \mathrm{~m}-$ long rovers, Spirit and Opportunity, each have a six-wheeled rocker-bogie suspension, a five degreeof-freedom arm called the Instrument Deployment Device (IDD) [2], four sets of stereoscopic cameras, three spectrometers, a microscopic imager, and a Rock Abrasion Tool (RAT) for cleaning and grinding rock surfaces.

Spirit landed at Gusev Crater on January 3, 2004. Opportunity, Spirit's sister rover, landed at Meridiani Planum on January 24, 2004, and is described in a separate paper which also gives more background on the rovers' mobility software [3]. Spirit's first images showed a rocky plain similar to the Pathfinder [4] and Viking landing sites. Images taken during descent by the Descent Image Motion Estimation System [5] showed several craters which enabled a rapid localization of Spirit within an orbital image from Mars Global Surveyor. The first twelve sols (Martian days) after landing constituted the Impact-to-Egress phase, which saw the deployment of the rover's solar arrays, sensor mast, and mobility mechanisms, followed by egress from the lander [6].

Since it can take up to 26 minutes for a signal from Earth to reach Mars (and vice-versa), direct teleoperation of the rovers is impractical. Power and line-of-sight constraints also prohibit continuous communication with the rovers. Instead, the rovers are controlled by command sequences sent every Martian morning. The sequences are executed over the course of the sol, with one or two communication passes for downlink (usually via the Mars Odyssey orbiter) during which images and other data are returned to Earth. The time available each sol for rover operations is thus affected by how much solar energy is available (a function of season, atmospheric conditions, dust on the solar array, and local terrain slope) and when the orbiter is within line-of-sight. The first 90 sols of operations used a 16-hour planning cycle with separate teams for each rover, but as the teams shifted onto a schedule based on a normal Earth work week rather than an overnight Mars workday, the planning cycle decreased to between 5 and 10 hours depending on the phasing of Earth and Mars days. (A Martian day is roughly 40 minutes longer than an Earth day.)

Spirit's mission can be divided into several campaigns characterized by differing science interests, destinations, terrains, and operational techniques. The first major objective was to reach the rim of nearby Bonneville Crater (Figure 1) in the hope of finding exposed outcrops of bedrock, but none were found. Spirit then began a $3 \mathrm{~km}$ trek across the rocky plains toward the Columbia Hills. Upon reaching the hills, Spirit investigated the West Spur of Husband Hill, the highest peak in the range, for several months while dealing with the approaching Martian winter. Since Spirit is in the southern hemisphere of Mars, more solar power is available if the rover and its horizontallymounted solar panels are tilted to the north; this dictated the rover's path along the north flanks of Husband Hill as it later traversed toward Cumberland Ridge, which overlooks the Tennessee Valley and joins the north face of Husband Hill. Fortuitously, high winds cleaned most of the dust of Spirit's solar panels on Sol 418. This eliminated the need to stay on north-facing slopes and opened up an easier route to Husband Hill's summit via the mountain's west face.

\section{Rover Mobility and Software}

Spirit's six wheel drive, four-wheel steering, and rocker-bogie suspension provide for excellent stability, maneuverability, and obstacle negotiation. The rover's ground clearance is $29 \mathrm{~cm}$, though operationally we treat rocks or other terrain features taller than $20 \mathrm{~cm}$ as obstacles. 


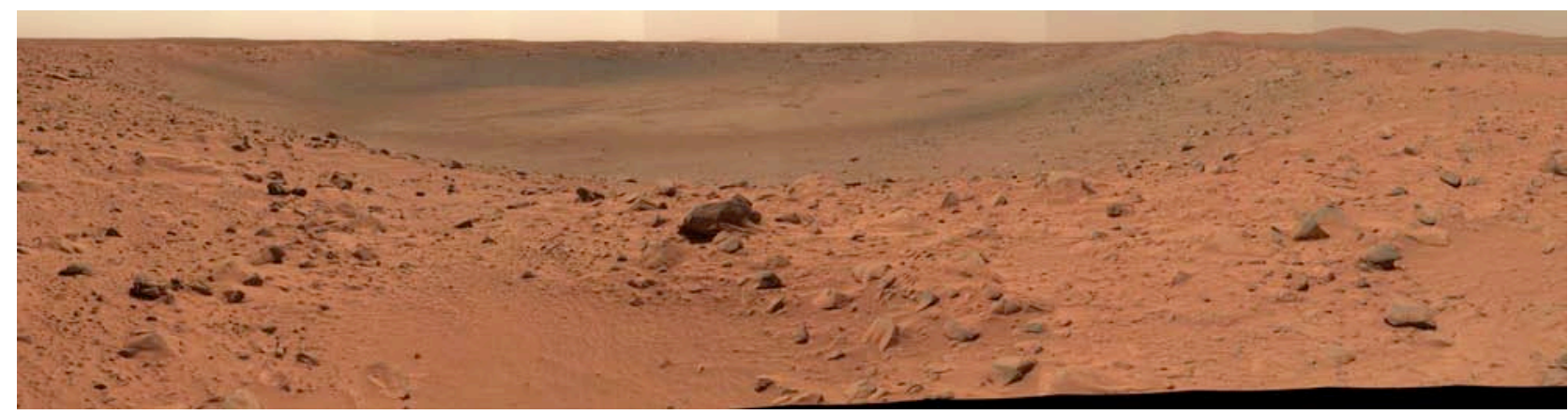

Figure 1: 200m-wide Bonneville Crater, with the Columbia Hills 3km away in the background. Husband Hill is the highest peak in the complex, standing roughly $90 \mathrm{~m}$ above the plains.

The rover's static tip-over angle is 45 degrees but slippage, rather than drive torque or stability, is usually the limiting factor when driving on slopes. Sandy slopes of as little as 10 degrees can completely block further progress, and on steep slopes with firmer footing, special techniques are required for safe and accurate driving.

Spirit's onboard sensors and software provide many mobility and safeguarding capabilities. The inertial measurement unit (IMU) produces roll, pitch and yaw measurements at $8 \mathrm{~Hz}$, and stereo camera pairs provide accurate position knowledge and terrain assessment. Reactive safety checks can halt vehicle motion if the suspension articulates beyond a preset limit, or if the pitch, roll, or overall tilt exceed the commanded range. The rover's command sequencing language also allows safety sequences to run in parallel with a drive and halt motion on other conditions, such as when the rover enters a manuallydefined keep-out zone.

When on level terrain, the combination of IMU and wheel odometry data leads to drive accuracy within a few percent. In terrain where slip is substantial and high accuracy is required--either to avoid obstacles or reach a desired location for science observations--visual odometry must be used, since the rover has no other way of detecting slip as the rover drives [7]. But visual odometry (VisOdom) is costly given Spirit's radiation-hardened $20 \mathrm{MHz}$ RAD6000 CPU, requiring roughly 3 minutes of processing for every $60 \mathrm{~cm}$ of commanded motion. Because of limited development time, VisOdom was originally viewed as a "nice-to-have" feature rather than a requirement. But VisOdom turned out to be essential on both rovers, which have spent most of their time on steep slopes due to the presence of scientifically valuable rock outcrops.

Spirit's software can also autonomously detect hazards based on imagery. The software analyzes stereo image pairs and assesses terrain safety based on obstacle size and overall slope. The rover has two modes for performing terrain assessment: AutoNav, which chooses paths based on terrain assessment to try to reach a specified location, and guarded moves, in which manually-specified motions can be vetoed by the rover if the terrain is not safe. Guarded moves limit the rover to a more predictable path, but are more brittle in the sense that an obstacle can cause the rest of a drive to be terminated. AutoNav can select its own path, which makes it able to deal with unforeseen hazards-with the limitation that non-geometric hazards such as loose soil are not detected. Like VisOdom, terrain assessment is quite costly in terms of execution time: each assessment takes about 3 minutes, and assessment must be performed every $50-150 \mathrm{~cm}$.

While the robotics research community has traditionally emphasized the path planning problem, most of Spirit's drive distance has relied on human analysis of imagery to identify obstacles and choose paths. Blind drives, which do not use obstacle avoidance and which use the IMU and odometry to follow a specified trajectory, have a speed of $130 \mathrm{~m} / \mathrm{hr}$. In very benign terrain, AutoNav can skip some imaging steps and achieve $35 \mathrm{~m} / \mathrm{hr}$. When only 1 to 3 hours are available for driving, it is most productive to drive blind as far as safely possible (within the limitations of terrain visibility and expected drive error) and then turn control over to AutoNav. When visual odometry is required, rover operators use sequencing techniques to specify keep-out zones around obstacles, and rely on VisOdom to compensate for drive errors and track the rover position well enough that motion can be halted if the rover enters a keep-out zone. Even if Spirit's CPU were fast enough to use VisOdom and AutoNav without an operational cost, significant further terrain assessment and adaptive driving capabilities would be required to safely guide the rover through steep terrain which no human or robot has ever seen before, and which in our experience can have unpredictable physical properties that are not deducible from imagery alone.

\section{Drive Sequencing}

Most drive sequences could be classified as either traverses (which cover maximum distance) or approaches (which drive to a specific position for subsequent arm operations). We quickly developed a template for traverse sols and used it for the first few months of the mission, when Spirit was driving on reasonably level ground with slopes of a few degrees. Our template consisted of a blind drive as far as the terrain and our imaging would safely allow, followed by an AutoNav drive until reaching a timeout called the Mobility Time-of-Day limit, so that Spirit could stop driving and begin image acquisition in time for the afternoon's communication pass. Since the amount of data returned is affected by the rover's heading, we often performed a turn to a specific heading to maximize data return. Each drive typically ended with acquisition of a "penultimate" Hazard Camera (HazCam [8]) stereo image pair, a 50-100 cm guarded arc, and an 


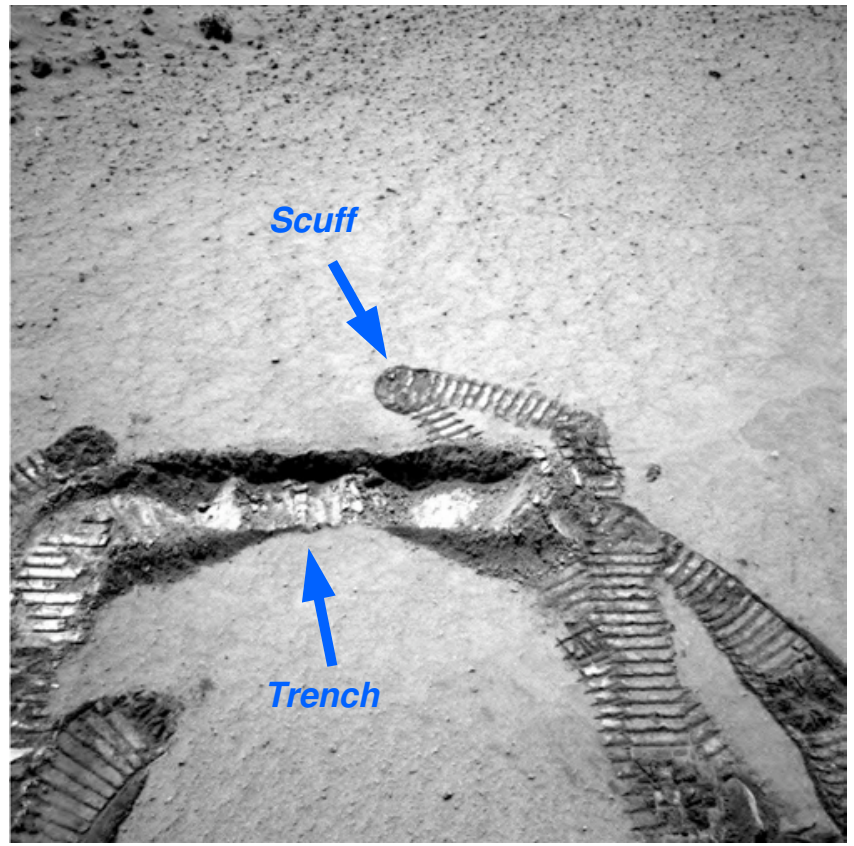

Figure 2: NavCam image of trench and scuff created on sol 47. The trench is up to $7 \mathrm{~cm}$ deep in places, and roughly $60 \mathrm{~cm}$ long.

"ultimate" HazCam pair at the rover's final position. The penultimate images allow the front HazCams to view the area below the rover's final position so that the IDD may be safely deployed. (The HazCams, which use fish-eye lenses, have a 180-degree diagonal field-of-view and are mounted roughly $50 \mathrm{~cm}$ above the ground)

Our early approach drives were typically under $2 \mathrm{~m}$ in length, primarily because the uniform nature of the rocks and soil rarely provided compelling reasons to alter our drive path. The instrument arm's workspace is roughly $40 \mathrm{~cm}$ long by $60 \mathrm{~cm}$ wide, and approach drives aimed to place the desired target within this volume. We planned conservative approaches, preferring to undershoot the target and then execute a short drive for fine positioning rather than end up with the target beneath the rover and out of the HazCam's field of view. We later used visual odometry and conditionally-executed commands to achieve fine positioning from 5-10m away even on steep slopes with high and variable slip.

\section{Sols 12-89: Bonneville Crater}

Spirit spent her first sols on the surface investigating a basaltic rock named Adirondack. The first drives were blind drives of 3 to $5 \mathrm{~m}$, and on Sol 36, a drive of $7 \mathrm{~m}$ was planned using both blind and AutoNav (autonomous navigation) drives. Early AutoNav drives exhibited an oversensitivity to changes in terrain slope, leading AutoNav to regard transitions between flat and sloped areas as hazards. Although this problem had been found and fixed during Earth-based field tests prior to landing, because it did not impact vehicle safety the decision was made to continue to use the more conservative pre-field test software for the duration of the three-month Primary Mission. As luck would have it, most of the terrain between the lander and Bonneville's rim was fairly level, and Spirit's highly accurate odometry (roughly 3\% accuracy on the plains) enabled us to confidently plan blind drives of increasing distance (up to $25 \mathrm{~m}$ en route to Bonneville).

Another repeated drive operation was "trenching", which used low-level motion commands to dig a $5-10 \mathrm{~cm}$ deep trench with one of the rover's front wheels. Spirit performed her first trench in "Laguna Hollow", then spent several sols inspecting subsurface materials with the instrument arm. We also sequenced occasional "scuffs" to disturb the surface soil and break up hard soil crusts.

After investigating several rocks with the full instrument suite, Spirit reached the final slope leading to the rim of Bonneville Crater on sol 66. The rock density had increased as we approached the rim, since larger rocks were not thrown as far as small ones by the impact that created Bonneville. A PanCam (color high resolution panoramic camera) image of the final slope is shown in Figure 3. We sequenced a blind drive to the horizon shown in the image, with an AutoNav drive following. AutoNav worked well, first backing off from an obstacle in its path before proceeding. Contrary to our fears, the rim of Bonneville was fairly broad and flat, though with many blocks and dunes, and several further drives were needed to reach the inner bowl of the crater. After spending several sols acquiring a color panorama of the crater (Figure 1), the Columbia Hills were identified as our next long-term target and Spirit began an easterly traverse of the crater rim. Along the way, we used Spirit's wheels to cut into several serpentine sand dunes, and used the Rock Abrasion Tool to brush and grind a rock dubbed "Mazatzl" (Figure 4). Spirit then began descending the rim toward the plains, and on sol 89 Spirit's total odometry was $617 \mathrm{~m}$--meeting one of the mission success criteria $(600 \mathrm{~m}$ of traverse) one sol before the primary mission ended.

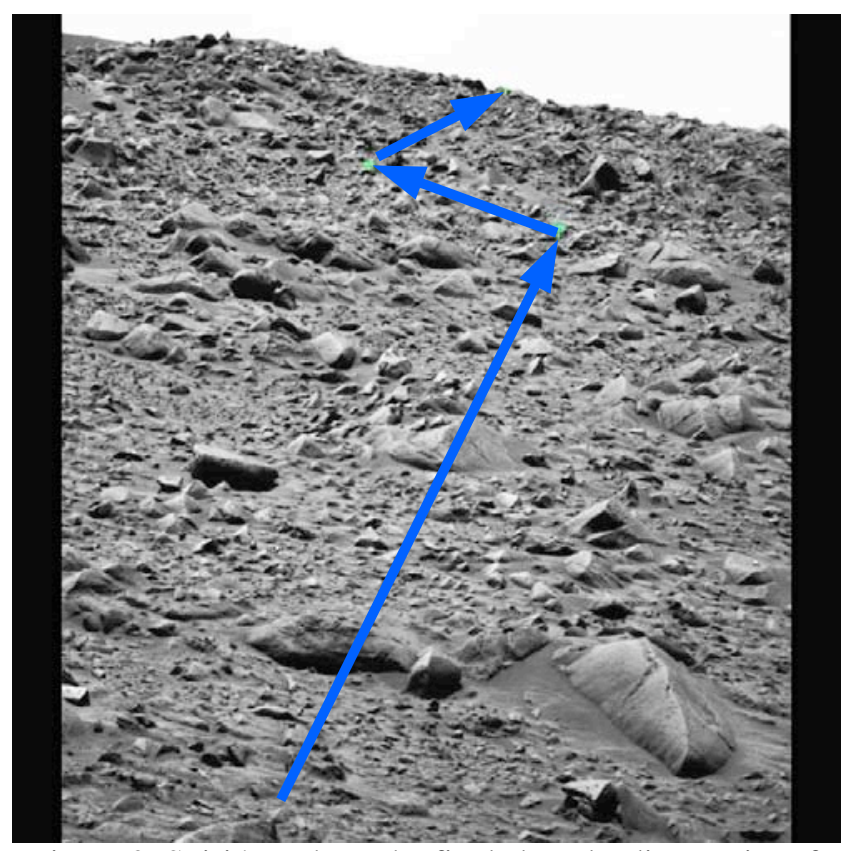

Figure 3: Spirit's path up the final slope leading to rim of Bonneville Crater. Average slope is 5 degrees, and the largest rocks are $25 \mathrm{~cm}$ tall. 


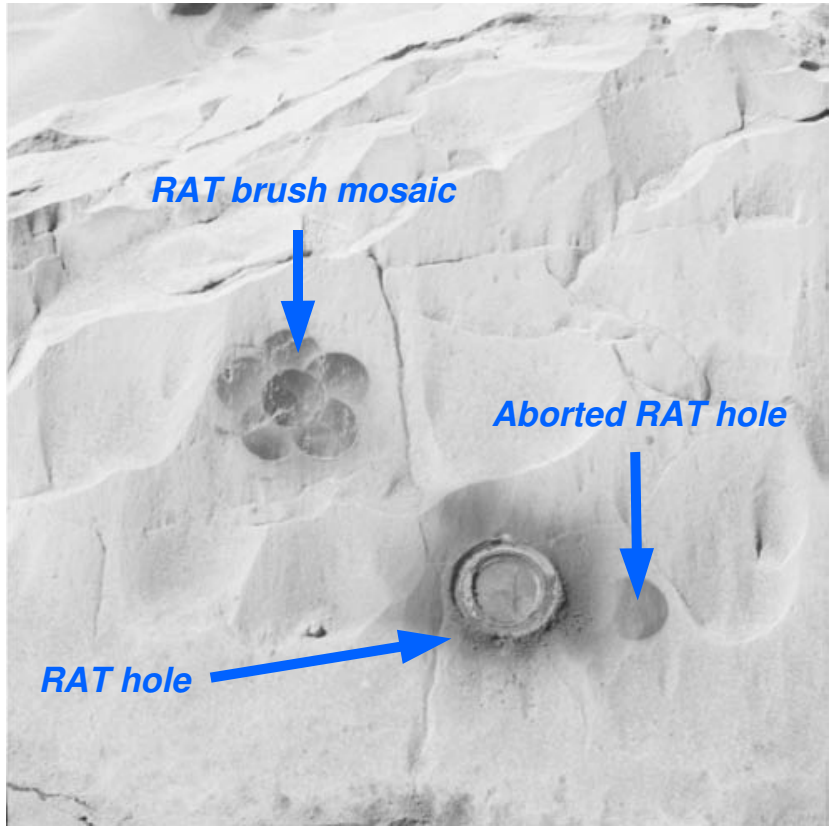

Figure 4: RAT brush mosaic and two RAT grind locations on Mazatzl. Individual RAT grind/brush locations are $5 \mathrm{~cm}$ in diameter.

\section{Sols 90-154: Crossing the Plains}

Having completed the investigation of Bonneville Crater, Spirit received new flight software designed to fix several bugs and improve mobility. The most important software changes for Spirit's near-term driving related to AutoNav: first, the obstacle assessment code was changed to prevent slope transitions from being seen as obstacles, and second, if the terrain in front of the rover was reasonably benign, then the rover would rely on the existing hazard assessment and skip taking new images for several steps--a crucial speedup given Spirit's slow processor.

Over the next 65 sols, Spirit's odometry reached 3.2 kilometers, with a total of 1.1 kilometers under autonomous control. The same traverse template (blind drive followed by AutoNav, then a turn for communications and penultimate/ultimate imagery) was used throughout the traverse to the Columbia Hills. We settled into a "Drive Sol Quartet", so named for the four sets of science observations performed on successive drive sols. The rationale behind the quartet was that a systematic set of observations was desirable to characterize the terrain over the duration of the traverse, but limited time and power precluded taking the full suite of observations during every drive. Only one sol template out of the four included the penultimate HazCam imagery, so that instrument arm operations were precluded for three out of four sols (during which Spirit instead performed PanCam, Mini-TES, and NavCam remote sensing). The time allocated for driving typically ranged between 1.5 and 2.5 hours per sol, depending on sol type. The routine drive pattern allowed us to refine our drive techniques, using 4 NavCam stereo pairs to cover close to 180 degrees of terrain up to $30 \mathrm{~m}$ away and 4 PanCam stereo pairs to cover roughly 45 degrees of terrain up to $70 \mathrm{~m}$ away. We applied the same hazard assessment software used by the rovers to build obstacle maps and used both image-based measuring tools in SAP [9] and terrain meshes in RSVP [10] to manually assess hazards and measure rock sizes. At 30 or more meters, even small height variations in the terrain resulted in occluded regions in which we had no terrain information. When these regions were $1 \mathrm{~m}$ or less in length, we deemed them safe to traverse based on the terrain we had seen. Larger occluded areas were regarded as obstacles, which we either had to avoid in our blind drives or cross using AutoNav.

The traverse rate with blind drives was roughly $130 \mathrm{~m} / \mathrm{hr}$, and in benign terrain AutoNav could achieve up to $35 \mathrm{~m} / \mathrm{hr}$. This speed differential pushed us to sequence the longest blind drives we felt were safe, up to $70 \mathrm{~m}$ though 40 to $50 \mathrm{~m}$ was more typical. Spirit's longest drive was $124 \mathrm{~m}$ on sol 125, and the longest AutoNav segment was $79 \mathrm{~m}$, on sol 133. Drive sequences typically used 2 to 5 blind waypoints and 1 or 2 AutoNav waypoints.

While our primary goal was to reach the Columbia Hills, we took several short detours to visit other craters. The first was Missoula Crater, which marked the first time we commanded a long AutoNav drive into terrain we had not yet seen. After reaching the rim of Missoula on sol 105, we commanded a blind drive of roughly $55 \mathrm{~m}$ to a point on the local horizon, after which AutoNav took Spirit down Missoula's rim toward the plains. AutoNav is conservative by design and had kept Spirit safe until that point, but it was still a nerve-wracking wait for the end-of-drive imagery. Post-drive NavCam images looking backward showed that AutoNav had correctly avoided several large rocks while negotiating a 10 degree slope (Figure 5).

On sol 120, we approached the rim of Lahontan crater. The drive to the rim showed unusual behavior during a Go_To_WAYPOINT command. As the rover approached the designated goal, it executed several anomalous back-andforth arc segments as it tried to get within $0.5 \mathrm{~m}$ of the goal. The cause of this was unknown at the time, though it later turned out to be a bug in flight software related to the elevation change between the goal and the origin of the most recently-defined local coordinate system.

On sol 148, Spirit's odometry surpassed 3 kilometers and the West Spur of Husband Hill grew near. Unfortunately, our rapid and trouble-free progress was not to last. By sol 154, the drive motor for the right front wheel had begun to draw over twice as much current as the other wheels, and the gentle terrain of the plains gave way to denser rock fields and $50-100 \mathrm{~cm}$ high hummocks. On sol 155 , we tripped the maximum tilt fault protection check when the rover's tilt exceeded 22 degrees as it climbed over a hummock. We increased the tilt limit for sol 156's drive, but after $40 \mathrm{~m}$ of driving, the drive faulted out as the rover tried unsuccessfully to reach a waypoint on a slope. Slippage induced a heading error that the rover was unable to overcome with its tightest allowable arc, and Spirit slipped further downhill as it repeatedly reversed direction while attempting to reach the goal. The path we had planned stayed clear of any obstacles, so rover safety was not compromised. 


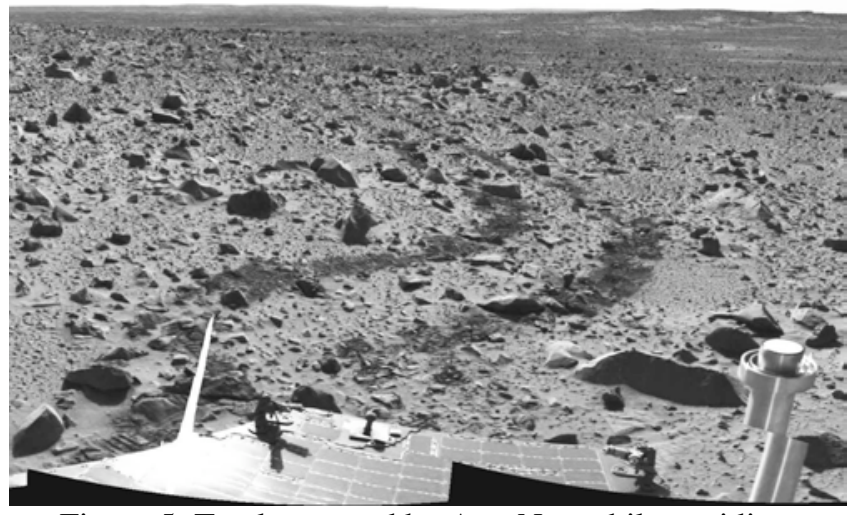

Figure 5: Tracks created by AutoNav while avoiding obstacles on a slope

\section{Sols 157-312: The West Spur}

As counterpoint to the difficulties we experienced in our final drives leading to the Columbia Hills, Spirit's new surroundings provided a wealth of science. Many nearby rocks had strikingly different morphology from the basaltic rocks we had seen on the plains, and observations with the instruments on the arm indicated a different geochemistry as well. The first target we studied in depth was a $10 \mathrm{~cm}$ rock named "Pot of Gold", and it revealed a uniquely Martian composition. After initial spectroscopy and imaging with the Microscopic Imager, we repositioned the rover to optimize the placement of the RAT on Pot of Gold. However, 10 to 13 degree slopes caused the repositioning to take several sols as the rover slipped up to $50 \%$ in loose sand. (We define slip as the reduction in distance traveled relative to commanded distance, so that $50 \%$ slip indicates the rover traveled only half as far as commanded and $100 \%$ slip indicates that the rover did not travel at all.) Dealing with slip continues to dominate our drive sequences as we climb Husband Hill.

After studying Pot of Gold, Spirit moved toward a relatively flat and level spot several meters away dubbed Engineering Flats, so named to reflect the engineeringdriven activities planned for the location. The drive to Engineering Flats tested Spirit's VisOdom software, which had already been used extensively on Opportunity. VisOdom allowed Spirit to measure its true motion by tracking features in NavCam images acquired every $0.6 \mathrm{~m}$ of commanded travel and automatically adjusting the rover's onboard position estimate, thus allowing accurate driving in high-slip areas. Once at the flats, Spirit's first task was to execute several short, low-speed drives designed to gather data on the right wheel's high current draw. Two sols of wheel actuator heating ensued in the hopes that heating might allow the viscous lubricant in the actuator's harmonic drive to redistribute itself more evenly. The wheel characterization drives were repeated after heating, but did not indicate an improvement and did not shed light on the source of the problem. Finally, Spirit performed several instrument arm placements on the soil, not to gather science data but to measure the end-to-end accuracy of the instrument positioning system. In the meantime, the science team had identified a promising outcrop of rock high on the hillside. However, a direct approach to the outcrop was not feasible. The hillside faced southwest, and with both rovers entering the southern hemisphere's winter, Spirit had to stay on slopes with a northerly tilt to prevent solar power from dipping dangerously low. We quickly adapted our image processing tools to provide color overlays for NavCam and PanCam images to clearly indicate which areas had significant northerly tilt [11], enabling us to maximize rover power by tilting sunward during and after drives.

While driving to the north face of the West Spur, Spirit began using a 5-wheel driving technique designed to minimize the use of the right front wheel, which we considered to be near the end of its usable life due to its high current draw. This required us to drive backward with the wheel disabled, overcommanding the other wheels and steering slightly to one side to compensate for the dragging right wheel since the high gear reduction prevented the wheel from freewheeling. Turn-in-place maneuvers were ineffectual with the right wheel disabled, so we created the "Tricky Drive" sequence which determined the relative heading toward the goal and then either drove straight or with left or right curving arcs. After successfully driving for several sols using only 5 wheels, Spirit soon reached slopes of more than 10 degrees on which 5-wheel driving could not make progress. Since we had to drag the disabled wheel rather than pushing it forward, the disabled wheel was on the downhill side of the rover as it drove uphill. This placed more load on the downhill wheels, including the disabled wheel, thus increasing resistance while decreasing traction on the uphill wheels. This forced us to resume driving with 6 wheels, and we soon encountered slopes up to 25 degrees, again tripping the tilt safety check. Continuing, we neared an outcrop named Clovis on sol 203, but ended up in a southward-facing hollow after traveling roughly $1 \mathrm{~m}$ further than planned. To climb out of the hollow, we sequenced a multi-leg drive using VisOdom, a pattern that would come to be standard for driving on steep slopes. This pattern executes a series of fixed length arcs conditionally, only driving further if the VisOdom-updated position indicates that the goal has not been reached. This "conditional overcommanding" allowed us to compensate for slip by commanding extra motion that would only be performed if the rover slipped. This was necessary because VisOdom was not integrated with the Go_To_WAYPOINT command in this version of the rover software.

Spirit flawlessly executed the VisOdom portion of the drive, placing it only $2 \mathrm{~m}$ from its goal, but in a final crossslope Go_To_WAYPOINT it encountered the slope-related bug, executing several erroneous movements and sliding away from the goal. Several sols of high-slip driving--over $100 \%$ slip in one case with tilts up to 30 degrees--were required to reach our position atop Clovis. Once on Clovis, we performed extensive instrument placements on the outcrop and acquired the "Cahokia" panorama (Figure 6).

Once work at Clovis was completed, Spirit continued uphill while maintaining a northerly tilt to maximize power. Several other rocks were investigated before solar conjunction, when Mars and Earth are on opposite sides of the sun and the sun's position interferes with communication. Several sols before conjunction, we drove 


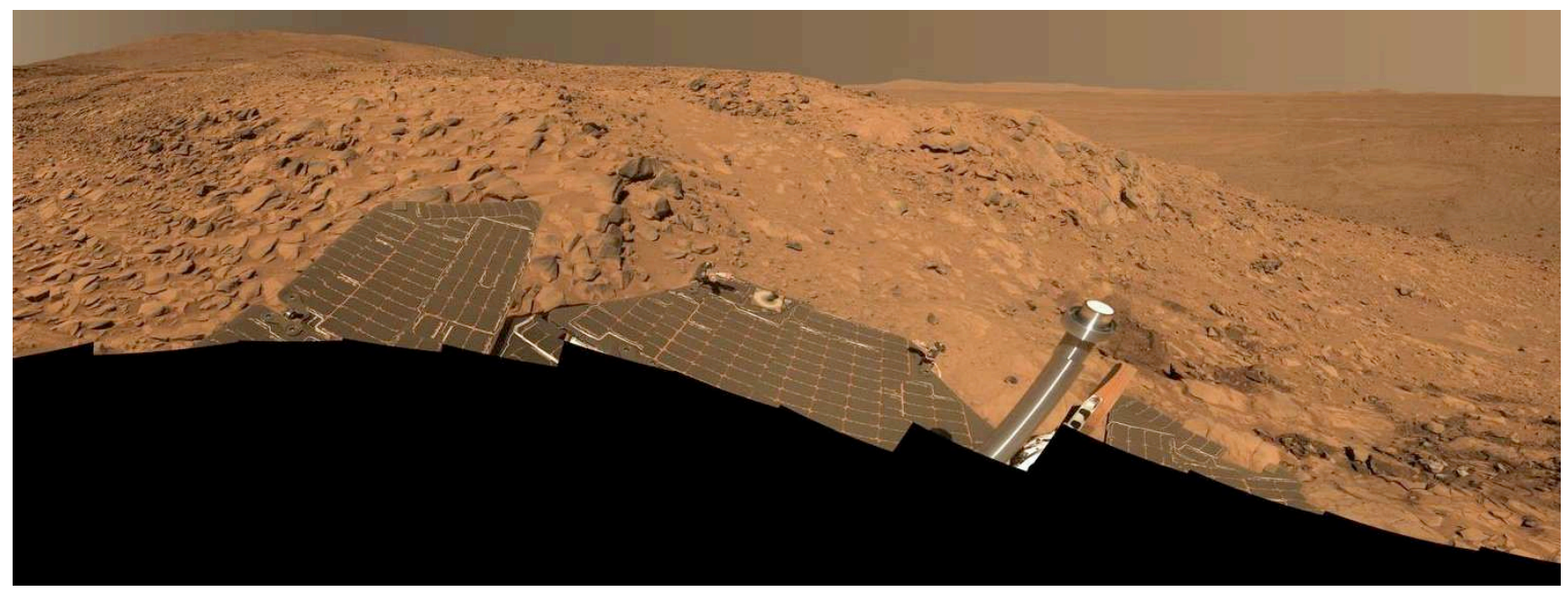

Figure 6: Part of the Cahokia Panorama, acquired starting on sol 210 while Spirit sat at a 21-degree tilt on the West Spur of Husband Hill. Cumberland Ridge and the summit of Husband Hill are visible on the skyline at left.

Spirit to a north-facing slope to provide good solar power. Spirit remained at this spot, dubbed Tikal, for sols 243-256.

Following conjunction, Spirit drove north toward a high point named Machu Picchu, with an ultimate goal of acquiring imagery to find a route to the summit of Husband Hill. Along the way, Spirit found a $10 \mathrm{~cm}$ layered rock named Tetl, and executed a short but complex approach drive using VisOdom and conditional sequencing. This was a refinement of previous drive techniques and used a series of small, conditionally-executed steps at the end of the drive to place Tetl squarely in the work volume, within $10 \mathrm{~cm}$ of the desired position despite the sloped terrain. A Microscopic Imager mosaic and multiple spectra were acquired at Tetl.

Spirit continued toward Machu Picchu, climbing slopes up to 20 degrees. On sol 312 we reached Machu Picchu and decided that the direct approach to Husband Hill was too risky due to terrain undulations, high slopes, large rocks, and the overall orientation of the hillside, which did not provide sufficient northerly tilt for solar power. We decided to traverse a broad saddle to the north face of Husband Hill, and on sol 312 drove off the West Spur and headed east.

\section{Sols 313-512: Cumberland Ridge}

Spirit's next goal was Cumberland Ridge, which we hoped would provide a north-facing route to the summit of Husband Hill and a view into the valley north of the summit. The descent from the West Spur and the initial traverse to the ridge provided a welcome rest from the technical and often 5-wheeled driving we had grown accustomed to. Slopes in the 5 to 7 degree range and a complete absence of obstacles allowed us to return to longer blind drives, though we were still limited by low power due to the Martian winter and gradual accumulation of dust on Spirit's solar arrays. In a stroke of good luck, short 6-wheel drives indicated that the right front wheel's drive current had returned to normal. The best explanation continues to be that infrequent driving and diurnal temperature cycles allowed lubricant to redistribute itself throughout the wheel's harmonic drive during 4 months on the West Spur.
With six functioning wheels, we quickly crossed the saddle to Cumberland Ridge but the easy driving was short lived: another slope-related software bug terminated our drive on sol 330, stranding Spirit in a sandy area with slips up to 95\%. After investigating a promising rock that happened to be in the arm's workspace, we attempted several uphill drives that did not yield any forward progress. By driving downhill and then cross-slope into less-sandy terrain, we made several meters of progress over two sols of driving, but these checks did not prevent further problems: on sol 343, Spirit's right rear wheel dug into loose material and engulfed a rock dubbed the Potato because of its size and shape (Figure 7). The rock jammed between the inner surface of the wheel and the housing of the steering actuator, stalling the drive motor. We successfully unwedged the rock, but it remained inside the wheel. For the next five sols, we reconstructed the situation in the testbed at JPL, building slopes and digging trenches to allow us to test strategies for ejecting the rock. We determined that we could safely drive the wheel roughly $1 / 6$ revolution in either direction, enabling small backward drives $(12 \mathrm{~cm})$ and left turns (7 degrees). Alternating between these two maneuvers gradually extracted the left rear wheel from the trench it had dug, eventually allowing the Potato to fall out of the wheel as it rotated on sol 346.

This experience gave us a new template for sequencing in which we overcommanded the rover to compensate for slip, but checked the rover's progress every meter or so using VisOdom to ensure that we had not encountered excessive slip--which could cause high sinkage and possibly another Potato incident. Although slippage was heavily dependent on slope and terrain type (bedrock, regolith, wind-blown sand or encrusted soil), it was also a function of how much the rover's wheels had sunk into the terrain. In soft soil, turn-in-place maneuvers contributed to sinkage since the wheels had to be steered substantially at the beginning and end of the turn. We realized we could minimize turn-in-place maneuvers by using a six-wheel version of the "Tricky drive" sequence which determined whether the goal was forward, slightly left or right, or further left or right, and chose either a straight drive, a 
curving arc, or a turn-in-place followed by a straight arc (in the case of large heading error). The Go_To_WAYPOINT command also used arcs and would have been appropriate, but it could not be used with VisOdom.

Since we had to use VisOdom for most drives, we could not afford additional time for hazard detection processing. On the other hand, with VisOdom we could be sure the rover would know where it was, which enabled us to specify keep-out zones surrounding known obstacles and halt motion if the rover strayed too close. Finally, we also developed a maneuver to extricate the rover from a "dug-in" position by executing a parallel parking maneuver, once in reverse and once moving forward. This maneuver results in a net sideways motion, with the intent of leaving all six wheels less dug in to allow further driving.

This combined set of drive techniques--visual odometry, conditional overcommanding, slip checks, keepout zones, and the Tricky Drive--allowed Spirit to perform safe and precise drives on slopes up to 20 degrees while tolerating slippage up to a preset limit (usually around $50 \%$ ). We usually had only 50-90 minutes for driving each sol, and with each $60 \mathrm{~cm}$ VisOdom step taking 2.5 minutes, our progress was usually between 10 and $15 \mathrm{~m}$. We used VisOdom whenever we anticipated high-slip terrain (essentially every day), but it proved extremely difficult to predict slip based on our imagery. For example, we saw slips of only $15-20 \%$ on a 19 deg slope, but 30 m further saw 95\% slip on only a $16 \mathrm{deg}$ slope. Color, high-resolution PanCam imagery of the region in which we had slipped did not show any difference in terrain appearance. In some terrain, slip can also vary widely (e.g. from $15 \%$ to $60 \%$ ) over successive $60 \mathrm{~cm}$ drive steps.

As one might imagine, the complexity of our drive sequences skyrocketed when incorporating all the techniques described above. Whereas a complete drive sequence for a 70 to $90 \mathrm{~m}$ drive on the plains typically had between 70 and 120 commands, our drive sequences on Cumberland Ridge had 200 to 500 commands for only 10 to $15 \mathrm{~m}$. However, these drives made extensive use of "helper sequences" (subroutines), so that the total number of commands expanded to 4000 for drives with 3 or 4 waypoints, and was over 1000 for even a $10 \mathrm{~m}$ drive with 1 waypoint. Additionally, these sequences had up to 50 conditional tests, which were originally intended to be used very sparingly and not for controlling major vehicle activities. It is worth noting that our planning timeline had shrunk from the original 16 hours during early operations to only 6-8 hours by later sols (keeping the planning to nominal working hours). Improvements in our sequencing tools and processes, along with a year of experience with the rover, allowed us to quickly build sequences far more complex than those used in the first months of the mission.

\section{Sols 513-535+: Husband Hill}

At time of writing, Spirit has survived over 535 sols on Mars and is climbing the western slope of Husband Hill using a flight software upgrade that was activated in March 2005. The new software has greatly simplified drive sequences by fixing slope-related bugs in the Go_To_WAYPOINT command and allowing us to use

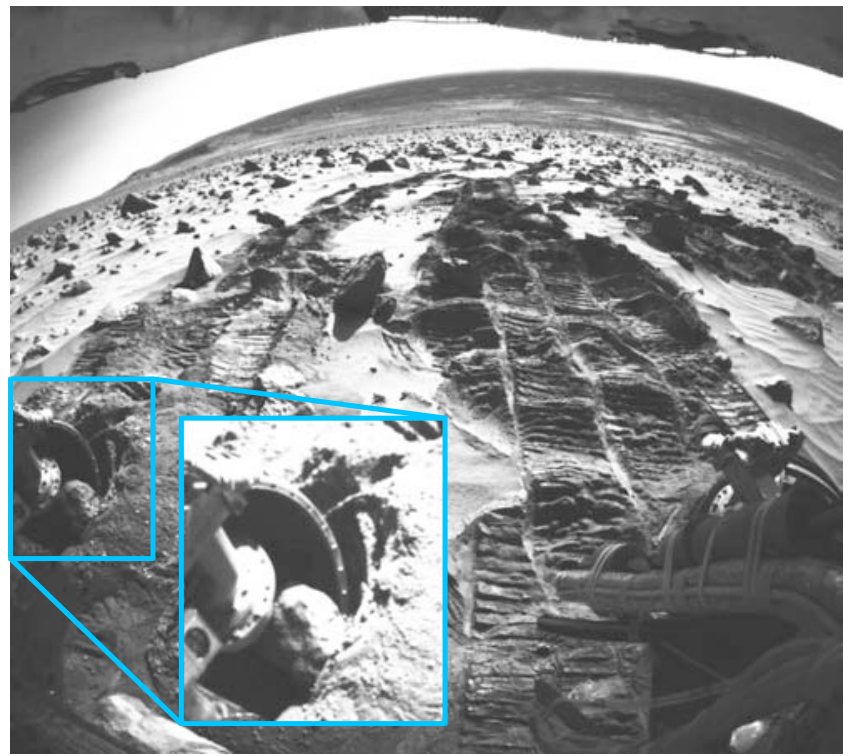

Figure 7: Rear HazCam image showing "Potato" rock stuck in the right rear wheel (inset). Note disturbed soil due to

high slip while driving diagonally up the sandy slope.

Go_To_WaypoInt with VisOdom. While Cumberland Ridge proved too difficult to climb due to a mix of 18 degree slopes, $25 \mathrm{~cm}$ rocks, and loose sand, the West Face has had much firmer terrain, though still steep and often rocky. We have been able to reliably drive 20 or more meters per sol using VisOdom drives, with one drive of 43 meters using VisOdom, blind driving, keep-out zones, and reactive sequencing that selects whether to continue using blind or VisOdom drives based on the amount of slip encountered. Spirit is currently $200 \mathrm{~m}$ from the summit of Husband Hill and has gained $60 \mathrm{~m}$ of elevation since arriving at the Columbia Hills.

\section{Conclusions}

Spirit's operation on Mars presented many unforeseen challenges. While most of Spirit's $4.5 \mathrm{~km}$ trek has been on level terrain, most of her drive sols and target approaches have been on slopes of 10 to 20 degrees. The need for safe, precise driving on steep slopes led to on-the-fly development of drive techniques, ground tools, and flight software updates. Our experience has made it clear that scientifically productive planetary robots can (and must) precisely navigate on slopes while meeting numerous constraints regarding obstacle avoidance, attitude, and heading. These constraints should be a strong driver in mobility configuration design for future machines targeted at exploration.

Due to MER's rapid development schedule (3 years from inception to launch), a vehicle for testing mobility software was not available until a few months before launch, and testing focused on basic mobility on level ground. Several subtle bugs remained in the code, and were only exposed once Spirit began driving on slopes. Geologists are drawn to steep terrain which can expose materials with varying origins and ages, but the extent to which the rovers would be operated on slopes was not anticipated. Once this became apparent, a 25-degree sloped 


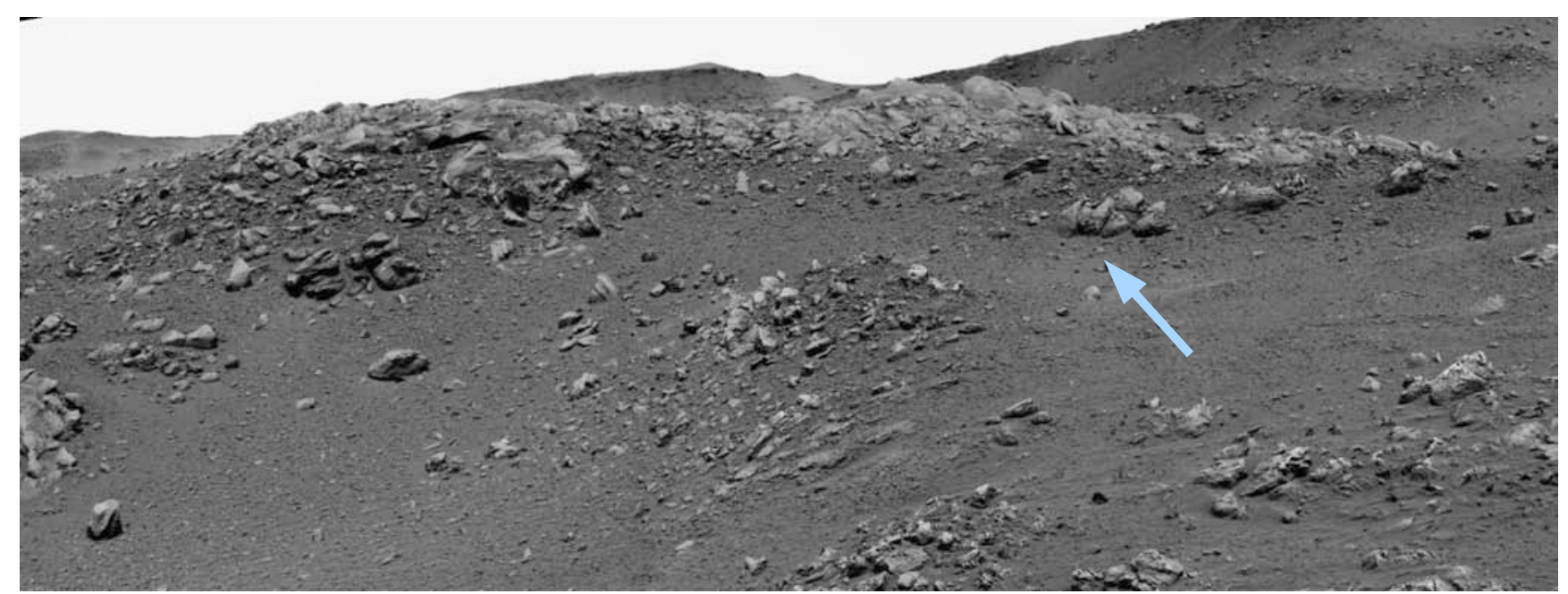

Figure 8: PanCam mosaic of Larry's Lookout, a high point on Cumberland Ridge. Slopes in the image range from 10-25 degrees; Spirit's location as of sol 406 is marked by the arrow. The slope at that point was 16 degrees. The pile of rocks directly above the arrow is roughly $30 \mathrm{~cm}$ tall.

platform was created for testing and development of new mobility software that was uploaded to the rovers after a year on the surface. Testing in realistic conditions is essential for robust performance.

Driving on slopes also led us to be heavily dependent on visual odometry. Once in the Columbia Hills (most of Spirit's mission thus far), we relied on VisOdom for nearly all of our driving. Even in terrain with $50 \%$ or higher slip, we were able to approach manipulator targets to within $10 \mathrm{~cm}$ of the desired position using VisOdom. Flexibility in the flight software and sequencing language was essential in allowing us to adapt to new terrain types and drive styles. Many parameters were adjusted to fine-tune sequences, and the availability of low, medium, and high-level behaviors was critical for safe, accurate, and effective driving in a range of terrains. Responsive ground software development was also key to efficient operation, with new tools often deployed within days of the identification of a need.

\section{Acknowledgments}

The research described in this paper was carried out at the Jet Propulsion Laboratory, California Institute of Technology, under a contract with the National Aeronautics and Space Administration. Thousands of people were involved in the mission at JPL and throughout the world, in government, academia, and industry. We gratefully acknowledge their outstanding work, which enabled us to explore Mars through Spirit and Opportunity. Finally, we would like to thank the citizens of the United States, whose tax dollars paid for this exploration.

\section{References}

[1] S. W. Squyres, et al. "The Spirit Rover's Athena Science Investigation at Gusev Crater, Mars". Science, Vol. 305, No. 5685, 6 August 2004.

[2] E. Baumgartner, R. Bonitz, J. Melko, L Shiraishi and C. Leger. "The Mars Exploration Rover Instrument Positioning System." In Proceedings of the 2005
IEEE Aerospace Conference, Big Sky, MT, March 2005.

[3] J. Biesiadecki, et al. "Mars Exploration Rover Surface Operations: Driving Opportunity at Meridiani Planum." In Proceedings of the 2005 IEEE Conference on Systems, Man, and Cybernetics.

[4] A. Mishkin, ,J. Morrison, T. Nguyen, H. Stone, B. Cooper, B. Wilcox, "Experiences with operations and autonomy of the Mars Pathfinder Microrover". In Proceedings of the 1998 IEEE Aerospace Conference, Aspen, CO, March 1998.

[5] Y. Cheng, et al. "The Mars Exploration Rovers Descent Image Motion Estimation System", IEEE Intelligent Systems, Vol. 19, No. 3, May/June 2004.

[6] K. Burke, et al. "12 Wheels on Mars - The Standup, Deployment, and Egress of the Mars Exploration Rovers", in Proceedings of the 2005 IEEE Conference on Systems, Man, and Cybernetics.

[7] Y. Cheng, M. Maimone and L. Matthies. "Visual Odometry on the Mars Exploration Rovers." In Proceedings of the 2005 IEEE Conference on Systems, Man, and Cybernetics.

[8] J. N. Maki, et al. "Mars Exploration Rover Engineering Cameras", Journal of Geophysical Research, Vol. 108, No. E12.

[9] P Backes, et al. "Sequence Planning for the FIDO Mars Rover Prototype." In Proceedings of the 2003 IEEE Aerospace Conference, Big Sky, MT.

[10] J. Yen, B. Cooper, F. Hartman, S. Maxwell, J. Wright, "Sequence Rehearsal and Validation on Surface Operations of the Mars Exploration Rovers." In Proceedings of SpaceOps 2004, Montreal, Canada, 2004.

[11] C. Leger, R. Deen, and R. Bonitz, "Remote Image Analysis for Mars Exploration Rover Mobility and Manipulation Operations." In Proceedings of the 2005 IEEE Conference on Systems, Man, and Cybernetics. 